\title{
The neurobiology of splitbrain crayfish
}

\author{
Baltazar Barrera-Mera ${ }^{1}$, Juan José Granados-Romero², José Juan Vargas-Morales ${ }^{1}$, \\ Elvira Barrera-Calva ${ }^{1}$, Rodrigo Banegas-Ruiz ${ }^{3}$, Emilio Pérez-Ortega ${ }^{1}$, \\ José Ángel Barajas Colón ${ }^{4}$, Francisco Fabian Gómez-Mendoza ${ }^{5}$, \\ Carlos Rubén Baca-Domínguez ${ }^{6}$, Yuri Jiménez Caprielova ${ }^{7}$, Román Alberto de la Rosa Llergo ${ }^{1}$, \\ Rodrigo Mendoza-Aceves ${ }^{8}$, Ana Lilia Abundez-Pliego ${ }^{9}$, Karen Uriarte-Ruiz ${ }^{1}$, \\ Mariana del Carmen Radilla-Flores ${ }^{10}$, Nelson Niels Espinosa Queb ${ }^{10}$, \\ Alejandro Ángel Corona Figueroa ${ }^{10}$, Bruno Andrés Salazar Trujillo ${ }^{10}$, \\ Alan I. Valderrama-Treviño ${ }^{11} *$
}

\author{
${ }^{1}$ Department of Physiology, ${ }^{2}$ Department of Surgery, Faculty of Medicine, UNAM, CDM, Mexico \\ ${ }^{3}$ Department of Traumatology, Rehabilitation Hospital, Luis Guillermo Ibarra, Ibarra \\ ${ }^{4}$ Department of Angiology and Vascular Surgery, CMNSXXI, CDMX, Mexico \\ ${ }^{5}$ Department of Hand Surgery, Central Military Hospital, CDMX, Mexico \\ ${ }^{6}$ Department of Hand Surgery and Microsurgery, Rehabilitation Hospital, Luis Guillermo Ibarra Ibarra, CDMX, Mexico \\ ${ }^{7}$ Department of Surgery, Angeles Villahermosa Hospital, Mexico \\ ${ }^{8}$ Department of Anesthesiology, Angeles del Pedregal Hospital, CDMX, Mexico \\ ${ }^{9}$ Department of Surgery, PEMEX General Hospital, Ciudad del Carmen, Mexico \\ ${ }^{10}$ Department of Plastic Surgery, CENIAQ, CDMX, Mexico \\ ${ }^{11}$ Department of Surgery, Central North Hospital PEMEX, CDMX, Mexico
}

Received: 15 March 2021

Revised: 25 March 2021

Accepted: 30 March 2021

*Correspondence:

Dr. Alan I. Valderrama-Treviño,

E-mail: alan_valderrama@hotmail.com

Copyright: (c) the author(s), publisher and licensee Medip Academy. This is an open-access article distributed under the terms of the Creative Commons Attribution Non-Commercial License, which permits unrestricted non-commercial use, distribution, and reproduction in any medium, provided the original work is properly cited.

\section{ABSTRACT}

Background: They crayfish brain is segmented into two symmetrical hemiganglia. Normally both hemiganglia are in direct communication through a series of well-defined neural bridges that cross de midline to form reciprocal cross connections.

Methods: An original study was carried out in the Department of Physiology of the Faculty of Medicine, UNAM during the period from August 2019 to August 2020. 13 Crayfish Procambarus clarkii weighing between $1 \mathrm{~g}$ to $30 \mathrm{~g}$ were used. Each eyestalk of the animals was tied to a displacement transducer coupled with a polygraph so that optomotor or electrical activity was bilaterally recorded. The separation of the right from the left hemiganglia from the cerebral or supraesophageal ganglion was performed with a sagittal section, splitbrain (SB).

Results: The normal photo motor reflex in crayfish eye is measured as a gradual decrease in the ERG amplitude. During tactile stimulation, the visual activation of both eyestalks in normal crayfish leads to a highly regular bilateral activity. The regular activity can only be altered by disturbing the mechanoreceptors located in the shell surrounding the eyestalks.

Conclusions: The procedure presented in this article provides unique characteristics for the study of the nervous system such as a detailed response of the bilateral optomotor reflex.

Keywords: Circadian rhythm, Electroretinography, Ganglia / physiology, Crayfish brain, Splitbrain crayfish, Circadian pacemakers 


\section{INTRODUCTION}

They crayfish brain is segmented into two symmetrical hemiganglia. ${ }^{1}$ Both of them containing a set of neurons and neuropil structures with the corresponding counterpart in each brain hemisphere. Normally right and left defined hemiganglia are in direct communication through a series of well-defined neural bridges that connect through the midline and form reciprocal cross connections between the corresponding structures of the left and right sides. For vertebrates and crustaceans, sensory information coming from photosensitive, chemical, and gravity motion, is processed by the cerebral and thorachoabdominal hemiganglia. This information is then organized and determines the endocrine and behavior response of the subject. $^{2}$

In this way, it is clear the importance of symmetry in visual, caudal photosensitivity and locomotive functions, and so this method offers a most suitable model to explore the significative role of bilateral integration upon corporeal activity in these crustaceans. For this purpose, we will explore the effects that occur when severing the midline neural bridges of cerebral hemiganglia upon visual, locomotion, feeding and mood in splitbrain (SB) crayfish.

\section{METHODS}

An original study was carried out in the Department of Physiology of the Faculty of Medicine, UNAM during the period from August 2019 to August 2020.

13 Crayfish Procambarus clarkii weighing between $1 \mathrm{~g}$ to $30 \mathrm{~g}$ were used. All of them were brought from their natural habitat.

During the experiments, the animals were: Kept in very similar conditions to their natural environment to measure the magnitude of escape reflex. Kept under controlled laboratory conditions; into medium size tanks of water 100- $60-8 \mathrm{~cm}$. Fastened by means of a cork glued with dental acrylic to the back of their shells.

During the recording time of their optomotor activity (9 to $12 \mathrm{am}$ ) or electroretinogram, each animal was kept in a 3.5 liters water container at $21^{\circ} \mathrm{C}$ under a continuous light environment. Each eyestalk of the animals was tied to a displacement transducer coupled with a polygraph so that optomotor or electrical activity was bilaterally recorded.

Tactile stimulation was applied, using a thin brush, to the eyestalk posterior receptor field in the crayfish carapace. The intensity and frequency of tactile stimuli were automatically controlled through an electronic device. The light stimuli were given periodically from six to fourteen days. Stimuli were applied with a small 222 Philips flashing bulb $(0.24 \mathrm{~A}, 2.2 \mathrm{~V})$ to the eyes. Separation of right and left hemiganglia from the cerebral or supraesophageal ganglion was successfully achieved in 8 animals using a sagittal cut (splitbrain). The first step was to deeply anesthetize the subjects by dropping them from $1.5 \mathrm{~m}$ into ice cold water, forcing a complete arresting state in the crayfish ranging from 10 to 15 minutes during they were completely immobilized. The supraesophageal ganglion was exposed through a small hole $(2.5 \mathrm{~mm}$ in diameter) in the anteromedial region of cephalotorax, in the basal portion of the rostrum. The stomach gastric camera was pushed back using a small piece of sterile cotton and a homemade stainless-steel knife $(2.5 \mathrm{~mm}$ length, $3.0 \mathrm{~mm}$ width and 100 um thick) was used to separate the hemiganglia. Then a small piece of chitin (2.5 $\mathrm{mm}$ ) was removed and kept in a Van Harreveld solution at $0^{\circ} \mathrm{C}$ until it was reimplated and glued on with acrylic cement to avoid any loss of haemolymph. The entire operation was performed under direct illumination of a Zeiss stereomicroscope. During the postoperative period, all animals accepted food and were cleaned from any residual preparation. Their opto and photomotor responses were examined. Only Animals with clear facets at the corneal surface, compound eye and strong optomotor responses were selected. This procedure demonstrated that when dividing the crayfish brain down the middle into its right and left halves, each cerebral hemi ganglion controls retinal sensitivity and the eyestalk movements in an independent manner from the contra lateral one.

\section{RESULTS}

The locomotive capacity was recorded by measuring the magnitude and displacement of the escape reflex (in centimeters), the amount of food consumed and grip strength were also recorded variants. The escape reflex was measured using both visual and tactile stimulation.

Total suppression of visual induction of the escape reflex was observed in splitbrain preparations while tactileinduced escape reflex was progressively recovered.

\section{Optomotor activity}

The structures that control eye movement are powerful elements, which stabilize significant features of the visual environment in the retina. For vertebrates and crustacean's sensory information is bilaterally working as the afferent portion of visual stabilization reflexes (Figure 1). Selected stimuli of the external word triggers interneurons that intentionally bring into focus certain specific areas of both compound eyes of the crayfish's eye movement. The importance of moto neural control that is responsible for movement of the crayfish eye is revealed when in the splitbrain crayfish the uncoupling of the hemispheres provokes incessant spontaneous and evoked optomotor activity. Normally during the resting phase of the locomotor and visual activity, the longer axis of both eyestalks of the crayfish maintains a $120^{\circ}$ angle between each other (Figure 1). Both eyes always show a simultaneous and equal amount of spontaneous movements from 3 to $10 \mathrm{sec}$ duration. When walking and after the splitbrain surgery, that interocular angle usually 
diminishes. In splitbrain crayfish the eyestalk preparation followed their own optomotor movement pattern without contra lateral influence. In comparison, during tactile stimulation, the visual activation of both eyestalks in normal crayfish leads to a highly regular bilateral activity.

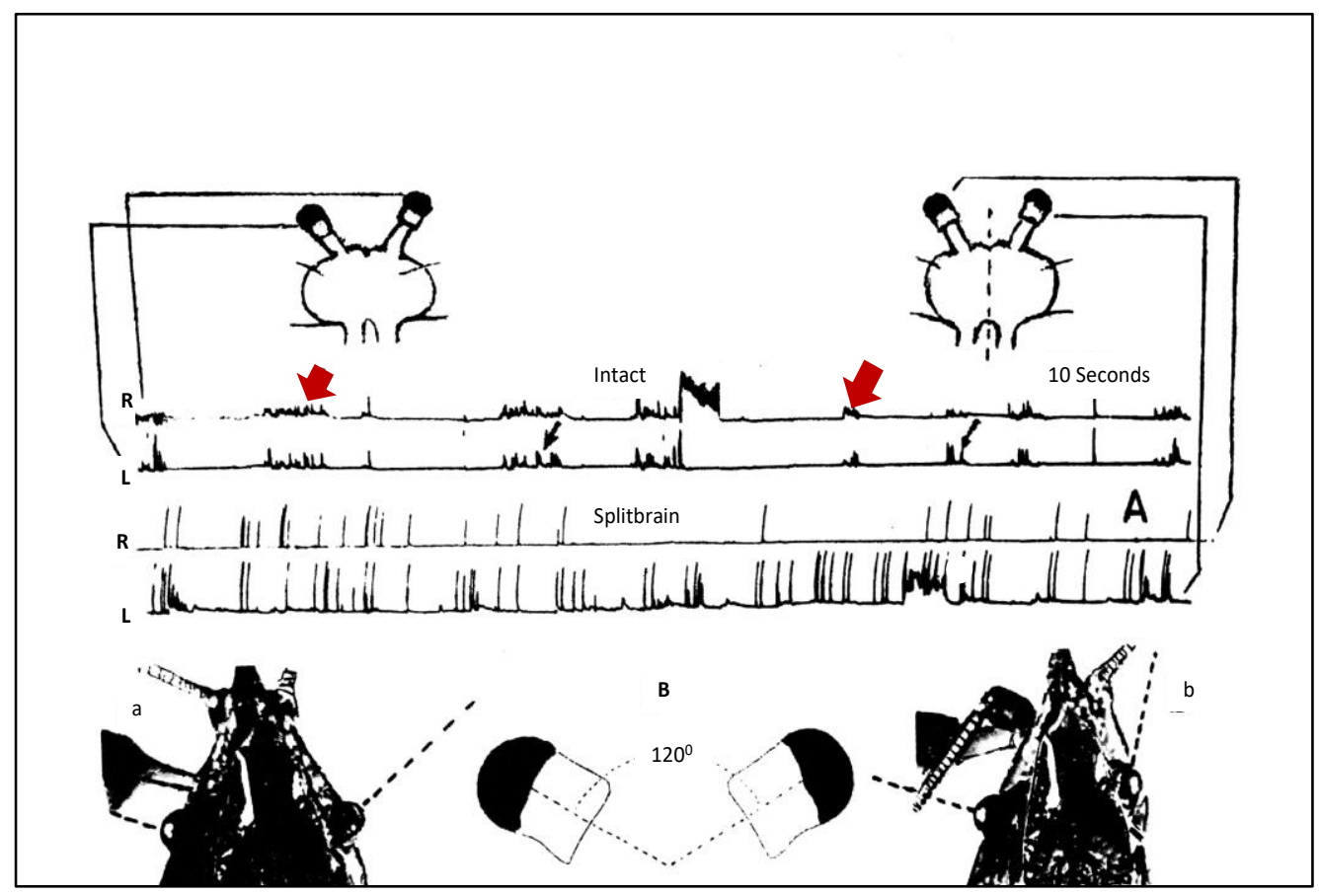

Figure 1: Bilateral response of the optomotor reflex. Effect of tactile stimulation (red arrow) applied to one eye stem in a healthy subject, observing bilateral optomotor response. Effect in bilateral spontaneous activity in a splitbrain subject. B. Normal angle of $120^{\circ}$ between eye stalks, a) Normal animal, b) animal with splitbrain.

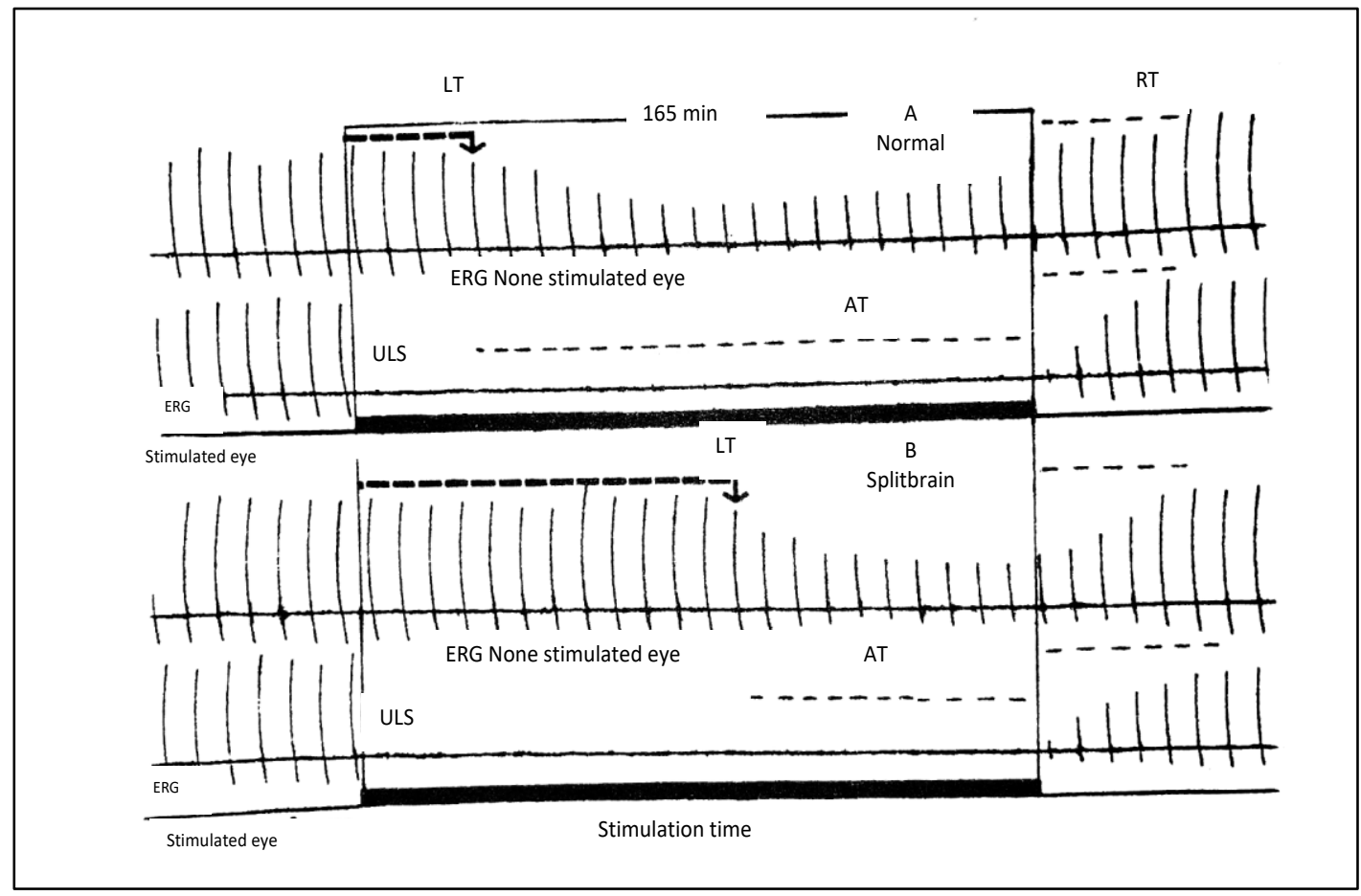

Figure 2: Electroretinogram (ERG). Normal animal (A) with unilateral light stimulation (ULS), note the consensual reflex on the contralateral side, with a short time of action (AT). Short latency time (LT). Prolonged latency time (80 minutes) in a splitbrain subject $(B)$. 


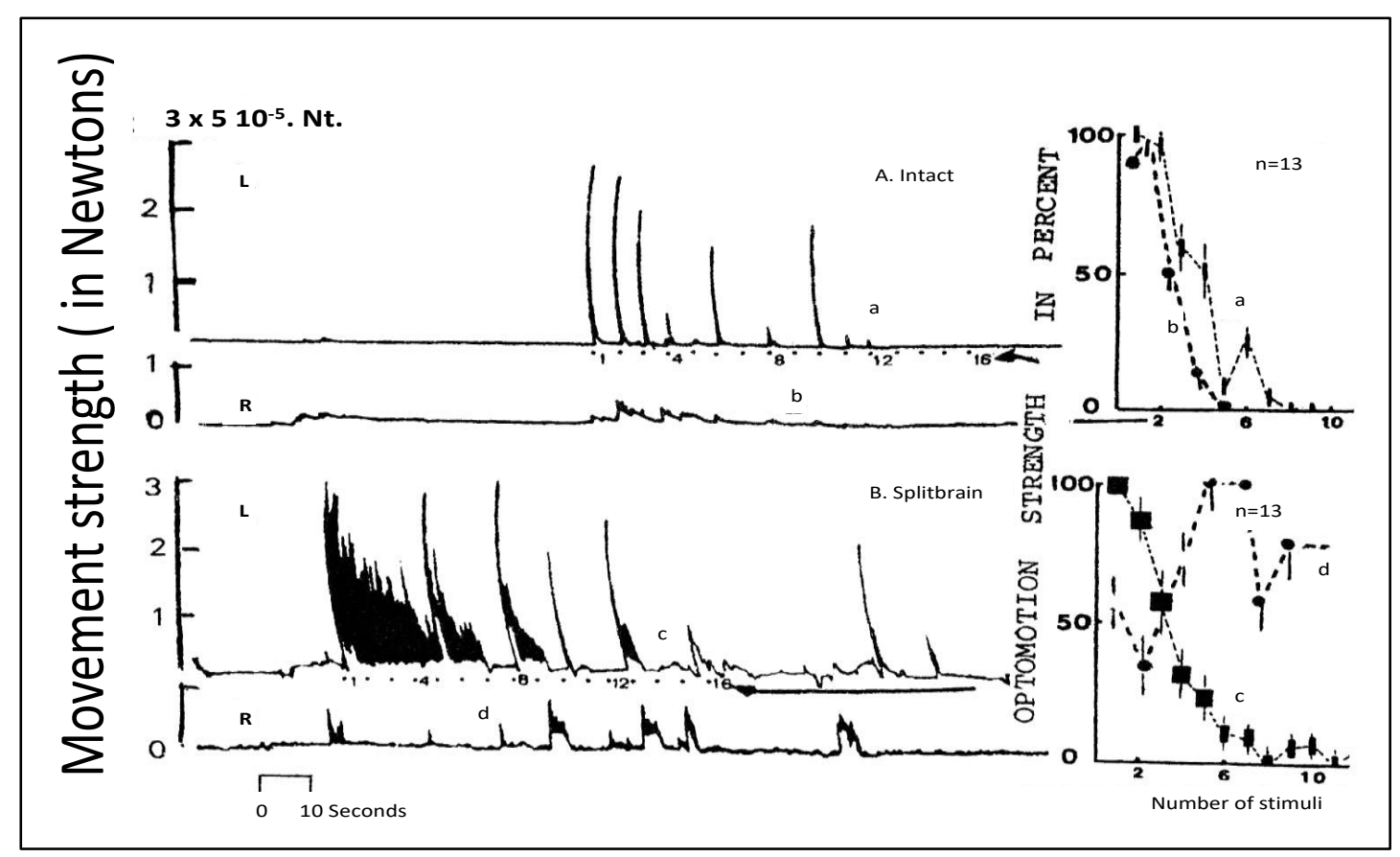

Figure 3: Habituation of the optomotor reaction. Normal animal (A) splitbrain subject (B). In the intact animal, bilateral habituation is observed, a. stimulated eye, b. contralateral eye. In the splitbrain animal, habituation occurs only in the stimulated (c.) eye. and is not seen in the (d.) contralateral eye.

\section{The consensual reflex}

The gradual decrease of electroretinogram (ERG) responses as well as the action time (AT) and the RT of retinal sensitivity were diminished compared to normal subjects (Figure 2). In both normal and SB crayfish, the total adaptation to light induced by sustained unilateral light stimulation (ULS) upon one single eye, was followed by a totally different darkness adaptation process in the contra lateral side. Another theory is that the light adapting hormone release of the ipsilateral stimulated ERG, required a typical consensual reflex as the one observed in normal animals (Figure 3).

\section{DISCUSSION}

Binocular focusing process makes possible the capturing of visual information accomplished by vestibular, visual, and proprioceptive modulation. ${ }^{3,4}$ The progressive recovery of retinal sensitivity started from zero amplitude of ERG response. ${ }^{5}$ Under the splitbrain (SB) conditions the progressive ERG diminution suggests the existence of a redundant contra lateral modulator influence whose signals travel throughout the circumesophageal connectives and probably also throughout subesophageal ganglion, the sustained ULS could be reaching the contra lateral side by using the sustaining fibers in these crustaceans. ${ }^{6,7}$

Throughout the specific participation of some protocerebrum interneurons, the redundant afferent signals running from crayfish retina connect ipsilateral optic lobes with their homologous counterpart. ${ }^{8}$ Besides their key role in visual perception, they revealed the presence of a potent neuroendocrine consensual reflex. This intricate phenomenon can be measure by means of both the light adapted retinal shielding pigments (RSP) position and also when measuring the diminishing amplitudes of the ERG. ${ }^{9}$ This, help us test the disruption of neuroendocrine bilateral integration in SB crayfish. The regular activity can be bilaterally broken only when disrupting the mechanoreceptors of the areas of the carapace sorrounding the eyestalks. In splitbrain conditions we can see the energic spasmodic movements of one single side. Unfortunately, the violent perpetual corporeal movements these subjects acquire from the active phase of the circadian activity of these animals totally mask the relatively feeble optomotor movements. The rate of the diurnal optomotor response was reduced after repeat tactile stimulation. Note that the non-stimulated or contralateral eyestalk also diminished its activity in a regular manner. The response rate first decreased and was totally absent after the stimuli were successively applied. They were becoming habituated. The identified groups of elements of oculomotor system of crayfish normally coordinating the conjugated movement of eyestalks, remains totally disconnected after the surgical bisection of crayfish brain, and with a large degree of independence.

We strongly consider the possibility that a powerful but reversible inhibitory influence acts simultaneously upon the left and right protocerebral circadian pacemakers while receiving bilateral photic stimulation. ${ }^{10}$ 


\section{CONCLUSION}

The model presented has unique characteristics for the study of the nervous system and proving very solid information about the bilateral optomotor reflex.

Funding: No funding sources Conflict of interest: None declared

Ethical approval: The study was approved by the Institutional Ethics Committee

\section{REFERENCES}

1. Smarandache CR, Wellmann. Arthropod neurons and nervous system. Current Biology. 2016;26(20):R960-5.

2. Barrera-Mera B, Abasta EM. Electrophysiological evidences of mutual modulatory influences on the retinal activity of the crayfish P. bouvieri. Brain Research Bull. 1978;3:101-10.

3. Barrera-Mera B. Neural coupling between left and right electroretinographic circadian oscillations in the crayfish. Comp. Biochem. Physiol. 1978;61(3):42732.

4. Kleinholz LH. Crustacean eyestalk hormone and retinal shielding pigment migration. Biol. Bull. mar. Biol. Lab. Woods Hole. 1936;70:150-84.

5. Bennitt R. Diurnal rhythm in the proximal pigment cells of the crayfish retina. Physiol Zool. 1932;5:659.
6. Barrera-Mera B, Cibrian-Tovar J, García-Díaz DE. The role of protocerebrum in the modulation of circadian rhythmicity in the crayfish visual system. Brain Res Bull. 1980;5(6):667-72.

7. Marguerite Webb HM, Miriam F, Bennett, Frank A, Brown Jr. A persistent diurnal rhythm of chromatophoric response in eyestalkless UCA pugilator. 1954;106:371-7.

8. Wiersma CAG, Yamaguchi T. The neuronal components of the optic nerve of the crayfish as studied by single unit analysis. J Comp Neurol. 1966;128:333-58.

9. Barrera-Mera B, Berdeja-García YG. Bilateral Effects on Retinal Shielding Pigments During Monocular Photic Stimulation in the Crayfish, Procambarus. J Experimental Biol. 1979;79:163-168.

10. Barrera-Mera B. Visual circadian rhythmicity in splitbrain crayfish: A plastic behavioral expression of symmetric circadian pacemakers. Brain Res Bulletin. 1985;15(2):203-8.

Cite this article as: Barrera-Mera B, GranadosRomero JJ, Vargas-Morales JJ, Barrera-Calva1 E, Banegas-Ruiz R, Pérez-Ortega E et al. The neurobiology of splitbrain crayfish. Int J Res Med Sci 2021;9:1276-80. 